\title{
INTELIGENCIA EMOCIONAL Y APEGO EN POBLACIÓN ADULTA: UNA REVISIÓN SISTEMÁTICA
}

\author{
Itziar Alonso-Arbiol, Ione Bretaña, Estibaliz Mateos-Pérez, Darío Páez, Bárbara Torres-Gómez, Aitzi- \\ ber Pascual, Susana Conejero y Mirian Gallarin \\ Universidad del País Vasco/Euskal Herriko Unibertsitatea, España
}

\section{Resumen}

La conceptualización de la inteligencia emocional (IE) y la teoría del apego en personas adultas constituyen dos de los marcos teóricos más relevantes para la comprensión del desarrollo socioemocional de los individuos y se originan de manera casi simultánea en 1990. Como consecuencia de ello, numerosos estudios afloraron para dar cuenta del influjo de las diferencias individuales en las diferentes dimensiones del apego y de la IE en diversas variables de personalidad y salud, y en ámbitos distintos (educativo, clínico, organizacional, etc.). En la presente revisión teórica se han examinado las investigaciones realizadas hasta la fecha que asocian medidas de ambos constructos con el objetivo de constatar su posible relación empírica. Así, se llevó a cabo una búsqueda sistemática en las bases de datos Web of Science, PsycArticles, PsycInfo y Psicodoc para localizar artículos de revistas revisados por pares, escritos en idioma castellano o inglés, que evaluasen de manera empírica la relación existente entre la IE y apego en población adulta. De un total inicial de 420 registros, tras las fases de escaneo y elegibilidad, fueron 25 los artículos analizados en el presente trabajo. En 24 artículos que describen estudios con metodología correlacional se constata la asociación empírica entre IE y apego, siendo esta positiva con respecto a la seguridad en el apego y negativa con las dos dimensiones de inseguridad. Los estudios se llevaron a cabo en 14 países diferentes y con muestras de población general, clínicas y de personas empleadas. Las correlaciones son mayores para la IE percibida que para las capacidades y para algunas dimensiones específicas. Se discuten las limitaciones y futuras líneas de trabajo en el ámbito emocional.

Palabras clave: Adultos, Apego, Inteligencia emocional, Revisión sistemática.

\begin{abstract}
The conceptualization of emotional intelligence (EI) and attachment theory in adults constitute two of the most relevant theoretical frameworks for understanding individuals' socio-emotional development and they originated almost simultaneously in 1990. As a consequence of this, numerous studies emerged to account for the influence of individual differences in the different dimensions of attachment and EI in various personality and health variables, and in different settings (educational, clinical, industrial/ organizational, etc.). In this theoretical review, we examined the investigations carried out to date that link measures of both constructs in order to verify their possible empirical relationship. A systematic search was carried out in the Web of Science, PsycArticles, PsycInfo and Psicodoc databases to locate peer-reviewed journal articles, written in Spanish or English, that empirically evaluated the relationship between EI and attachment in the adult population. From an initial amount of 420 records, after the scanning and eligibility phases, 25 articles were eventually analyzed in the present work. In 24 articles that describe studies with correlational methodology, the empirical association between EI and attachment is found, this being positive with respect to attachment security and negative with the two dimensions of insecurity. The studies were carried out in 14 different countries and with samples from the general population, clinical settings, and employed persons. The correlations were higher for perceived EI than for abilities and for some specific dimensions. Limitations and future lines of work in the emotional sphere are discussed.
\end{abstract}

Keywords: Adults, Attachment, Emotional intelligence, Systematic review.

Correspondencia.

\author{
Itziar Alonso-Arbiol \\ itziar.alonso@ehu.eus
}

Facultad de Psicología, Universidad del País Vasco UPV/EHU, Avenida de Tolosa, 70, 20018 San Sebastián

Este artículo se ha elaborado con la financiación de Investigación del Gobierno Vasco al Grupo "Cultura, Cognición y Emoción" (IT-1187-19). 


\section{Introducción}

En la última década del siglo XX se gestaron dos de los marcos teóricos más relevantes para la comprensión del desarrollo socioemocional de los individuos: la conceptualización de la inteligencia emocional (IE) y la teoría del apego. Por un lado, en 1990 se publicó el célebre artículo germinal en el que Salovey y Mayer acuñaron el término 'Inteligencia Emocional' para referirse a la capacidad del individuo para atender y distinguir los sentimientos y emociones propios y de otras personas, así como para utilizar dicha información para guiarse en la acción y pensamientos propios. Ese mismo año Bartholomew (1990) publicó el artículo que trasladaría los cuatro estilos de apego en la infancia a la adultez, y que sentaría las bases para el futuro estudio de las dimensiones del apego. Se cumplen, por tanto, 30 años de dos marcos de trabajo que han generado un ingente corpus de conocimiento.

En este artículo desarrollaremos una revisión sistemática teórica que dé cuenta de los estudios que han testado de manera empírica las interrelaciones entre ambos conceptos. Previamente, introduciremos a la persona lectora en los fundamentos teóricos y de la evaluación del apego adulto, así como en los preceptos conceptuales que relacionan apego e IE. Habida cuenta de que otros artículos incluidos en este monográfico ya han abordado en profundidad el concepto y los avances en el estudio de la IE, en el presente trabajo nos limitaremos a mencionar la distinción general entre los instrumentos de evaluación fundamentales como base-recordatorio para entender la revisión propuesta.

\section{Apego Adulto: Concepto y Evaluación}

El origen de la teoría del apego surge en el contexto de la infancia. El apego se definió inicialmente como un vínculo emocional particular que se forma entre el o la infante y su principal persona cuidadora (figura de apego) con la finalidad de otorgarle protección y un sentimiento de seguridad (Cassidy, 2008). Las conductas de apego responden a la necesidad biológica del bebé de lograr la cercanía, particularmente en momentos de malestar, con sus personas cuidadoras. Esta proximidad, además de favorecer su supervivencia, le ayuda a recuperar la estabilidad y el equilibrio psicológico que le proporciona la seguridad necesaria para explorar su mundo físico, emocional y social (Gregory et al., 2019). Aunque la teoría del apego ha sido el principal marco teórico para entender las relaciones entre infantes y cuidadores durante muchos años, también ha llegado a ser fundamental para comprender la personalidad y las relaciones íntimas en la etapa adulta. De hecho, ya Bowlby señaló que el apego caracterizaba el comportamiento y la experiencia de los seres humanos "desde que nacemos hasta que morimos" (Bowlby, 1969/1982, p. 208), siendo uno de los postulados esenciales de la teoría que las experiencias sociales tempranas influyen en la calidad de las relaciones íntimas posteriores (Feeney y Noller, 2001).
En este contexto, Hazan y Shaver (1987) plantearon que el vínculo emocional que se desarrolla entre los miembros de una pareja romántica es, en parte, una función del mismo sistema comportamental de apego que da origen al lazo emocional entre el bebé y sus cuidadores. Una de las principales contribuciones de su trabajo fue la idea de que el mismo tipo de diferencias individuales en el apego que se observan en los infantes (p.ej., seguro, ansiosoambivalente, evitativo) caracterizan también la manera en la que los adultos se aproximan a las relaciones íntimas. Por ello, adoptaron inicialmente el sistema de tres categorías de apego planteado por Ainsworth et al. (1978) como el marco para organizar las diferencias individuales en las formas en las que las personas adultas piensan, sienten y se comportan en las relaciones románticas. Así desarrollaron breves descripciones de los tres tipos de apego propuestos, solicitando a personas que, pensando en sus relaciones románticas, eligieran la descripción que mejor capturaba la manera general en que pensaban, sentían y se comportaban en tales relaciones.

Poco después, Bartholomew (1990) puso en cuestión la adecuación del modelo de tres categorías en el caso de las diferencias individuales en el apego adulto y lo reformuló. La autora defendía que las personas mantienen modelos representacionales separados de sí mismas (modelos del self) y de su mundo social (modelo de las otras personas) con diferentes implicaciones para la forma en la que la conducta de apego se organiza en la adultez. Dado que cada uno de estos modelos puede tener una valencia positiva o negativa, es posible derivar cuatro y no tres patrones principales de apego: el patrón seguro estaría caracterizado por una visión positiva del self y de los otros, el patrón inseguro-preocupado por una visión negativa del sí mismo y positiva de los otros, el patrón inseguro-evitativo se caracterizaría por una visión positiva del self y un modelo negativo de los otros, mientras que el patrón inseguro-temeroso lo estaría por una visión negativa de ambos modelos (del self y de los otros). Bartholomew y Horowitz (1991) desarrollaron el 'Cuestionario de Relación' (RQ), un instrumento conformado por cuatro párrafos que contienen descripciones breves de cada uno de estos tipos teóricos y que fue adaptado al castellano en población española por Alonso-Arbiol et al. (2007). Al igual que en la medida de elección forzosa de Hazan y Shaver (1987), las personas encuestadas debían elegir el párrafo que mejor recogiera la manera en que encaraban las relaciones íntimas.

Aunque a mediados de la década de los 90 parecía existir un consenso en la literatura acerca de que el modelo de cuatro categorías era el más apropiado para capturar las diferencias individuales en el apego adulto, los resultados de la investigación empezaron a desafiar varios de los postulados del propio sistema categórico: las clasificaciones no eran muy estables, eran mutuamente exclusivas y no tenían 
en cuenta la variación dentro de cada categoría. Como resultado de estos problemas, algunos investigadores (p.ej., Collins y Read, 1990) empezaron a solicitar a los participantes que evaluaran una serie de ítems de manera continua y a utilizar dichas evaluaciones para situar a las personas en un espacio multidimensional. Sin embargo, tal y como indicaron Gillath et al. (2016), surgieron dos problemas rápidamente. En primer lugar, no existía una razón fundamentada para pasar de una evaluación categorial a una continua, y mientras algunos investigadores utilizaban las puntuaciones para situar a los respondientes en varias dimensiones, otros las empleaban como un medio para obtener clasificaciones más precisas. En segundo lugar, la cantidad de escalas de auto-informe que se estaban desarrollando crecía sin límites, $\mathrm{y}$ el número de dimensiones recogidas en las mismas era muy variado (desde dos hasta siete). El trabajo de Fraley y Waller (1998) supuso un avance importante para apoyar la evaluación continua del apego adulto, ya que su análisis taxométrico arrojó resultados que eran más consistentes con una distribución continua de las diferencias individuales en el apego que con el modelo categorial. De hecho, aunque el sistema dimensional recoge los mismos patrones de apego que el sistema categórico original, posibilita que estos patrones sean representados con mayor grado de especificidad y fiabilidad de la que permiten los sistemas clasificatorios (Gillath et al., 2016). No obstante, la pregunta que surgía entonces era cuál era el mejor sistema dimensional para conceptualizar las variaciones en el apego adulto. Brennan et al. (1998) recogieron todas las medidas de auto-informe de apego adulto existentes en ese momento y las administraron a una muestra elevada para llevar a cabo un análisis factorial de las respuestas. Este reveló dos factores principales, denominados "ansiedad acerca de las relaciones" y "evitación de la intimidad", que de alguna manera se asemejaban a las dos dimensiones (modelo del self y modelo de los otros) que subyacen a los cuatro estilos de apego descritos por Bartholomew (1990). Siguiendo a Gillath et al. (2016), puede decirse que este análisis mostró que las diferentes medidas de apego adulto estaban recogiendo estas dos dimensiones básicas, al tiempo que permitió el desarrollo de un nuevo cuestionario: 'Experiencias en Relaciones de Pareja' - Experiences in Close Relationships (ECR) - , que ha llegado a ser la medida de autoinforme más empleada en la actualidad para evaluar las dimensiones de apego adulto y la cual también se ha adaptado a población española (Alonso-Arbiol et al., 2007). Las personas con puntuación alta en la dimensión de evitación pueden describirse como aquellas que necesitan sentirse más independientes y con mayor distancia emocional de la pareja para sentirse cómodas, mientras que las personas con altas puntuaciones en la dimensión de ansiedad se caracterizarían por una preocupación y un miedo excesivos a ser abandonadas por sus parejas.
No obstante, y más allá del contexto de las relaciones de pareja donde se originó, el apego adulto se ha mostrado como un marco eficaz en la comprensión de diferencias individuales en otras áreas (para una revisión, ver Mikulincer y Shaver, 2007). Así, por citar otro ejemplo, en el ámbito clínico son numerosos los estudios que dan cuenta de sintomatología psicopatológica afectiva a partir de las dimensiones de apego (p.ej., ver el metaanálisis de Woodhouse et al., 2015, sobre síntomas de estrés postraumático, o el de Zheng et al., 2020, sobre síntomas de depresión). Todo este entramado de relaciones puede explicarse por la naturaleza cognitivo-afectiva del vínculo afectivo. Desde este prisma, cobra sentido su relación con el desarrollo de capacidades emocionales, por lo que la convergencia de las dimensiones (o estilos) de apego y la IE podría postularse.

\section{Relación Conceptual entre Apego e Inteligencia}

\section{Emocional}

La teoría del apego comparte con la conceptualización de la IE el planteamiento de una base cognitiva que guía el procesamiento afectivo. La percepción emocional, el procesamiento emocional, la comprensión emocional y la regulación emocional son cuatro dimensiones o aspectos claves en ambas; constituyen las cuatro capacidades de la IE (Extremera et al., 2006; Mayer et al., 2002), así como los elementos que sustentan la variabilidad interindividual en el apego.

De esta manera, volviendo al esquema planteado por Bartholomew (1990), las diferencias en el apego se representan a través de la posición del individuo en cada una de las dos dimensiones-eje: "modelo del self" y "modelo del otro". Estos ejes implican un andamiaje cognitivo-afectivo o una teoría afectiva de cómo interpretar las respuestas de los demás; constituyen los denominados modelos operativos internos (internal working models) y funcionan como esquemas mentales bastantes resistentes al cambio (West y Sheldon-Keller, 1994). Tales esquemas condicionan la percepción de contenidos de tipo afectivo, así como las expectativas e interpretación de los comportamientos de personas cercanas emocionalmente. Asimismo, los modelos operativos internos guían el procesamiento de las emociones buscando su encaje o congruencia con la visión del self y de los otros, propias de cada estilo de apego. Una adecuada comprensión de las emociones es promovida en la comunicación emocional abierta entre las personas cuidadoras y el niño o niña que caracteriza a las interacciones que desembocarán en un apego seguro. Finalmente, el aspecto del apego más notablemente ligado a las capacidades del IE es la regulación emocional; de hecho, Bowlby (1969/1982) ya se refería a ella como una función primordial en la primera conceptualización de la teoría del apego en la etapa infantil. Cada tipo, dimensión u orienta- 
ción de apego se caracteriza por unas estrategias propias de regulación emocional. Las estrategias de hiperactivación (ansiedad) y desactivación (evitación) son formas de regularse emocionalmente que individuos con apegos inseguros han incorporado como consecuencia de una interacción temprana con personas cuidadoras no disponibles o con patrón de cuidados inconsistentes (Mikulincer y Shaver, 2016). Si bien en aquel contexto fueron respuestas adaptativas, la perpetuación de esa forma de regulación emocional y la focalización excesiva o la supresión de la emoción, ya en la época adulta y con personas disponibles emocionalmente, supondría una respuesta de regulación emocional no adaptativa (Mikulincer y Shaver, 2019).

Los individuos seguros se caracterizan por su apertura al mundo emocional, mostrando una percepción adecuada, no defensiva, de las emociones propias y ajenas. En cambio, las personas con un estilo o dimensión de apego inseguro muestran estrategias secundarias de naturaleza defensiva, ya que ha quedado inscrito en sus modelos operativos internos que el contacto con sus necesidades de apego insatisfechas les genera malestar (Mikulincer y Shaver, 2019). Así, tanto los evitativos como los ansiosos tienden a presentar dificultades en la percepción del dominio emocional en sí mismos y en los otros, mostrando déficits en la decodificación de los estímulos emocionales. Por otra parte, el procesamiento de la información emocional también se ve afectado de manera diferencial según el estilo de apego: las personas con una orientación del apego evitativa tienden a inhibir el procesamiento de los estímulos emocionales, mientras que los individuos con una orientación del apego ansiosa muestran una tendencia a la hipervigilancia de tales estímulos. De hecho, existe evidencia meta-analítica reciente que identifica los correlatos neuronales de tales modos de procesamiento emocional (Ran y Zhang, 2018). Con respecto a la regulación emocional, las personas con una orientación de apego evitativo intentan inhibir cualquier estado emocional que no sea congruente con su objetivo principal de mantener apagadas sus necesidades de apego (Mikulincer y Shaver, 2016). Aquí se incluyen fundamentalmente las emociones negativas. Este esfuerzo regulatorio, a su vez, les puede inducir a evitar el reconocimiento de las propias emociones. Además, para eludir la experiencia negativa de las emociones, la propia supresión retroalimenta la dificultad para incorporarlas en sus estructuras cognitivo-afectivas; en consecuencia, se limita el posterior procesamiento de información emocional y el comportamiento en un contexto interpersonal (Mikulincer y Shaver, 2019). La regulación emocional de las personas con orientación de apego ansioso, opera bajo esquemas de hiperactivación; la percepción de amenaza (del abandono) está aumentada, al igual que sucede con la percepción de las emociones negativas y del malestar asociado. En torno a esa amenaza se activa una hipervigilancia y atención a señales internas de contenido emocional negativo, incluyendo aspectos psicofisiológicos y cognitivos de rumiación (Cassidy y Kobak, 1988).

Resulta obvio, por tanto, que las capacidades de IE muestran paralelismos con el desarrollo socioemocional que subyace a la vinculación afectiva que se establece mucho antes de llegar a la adultez. Atendiendo a esta realidad conceptual, un número considerable de investigaciones han examinado los puentes tejidos entre la teorización de la IE y la del apego a lo largo de estos 30 años. Con el objeto de tener un acercamiento a los resultados de los estudios llevados a cabo en esta línea, en este artículo se presenta una revisión sistemática de los mismos. Si bien en este apartado se ha aludido a las capacidades de IE, una perspectiva igualmente fructífera está representada por la investigación de la IE percibida (Fernández-Berrocal et al., 2004). Así pues, se abordan en este trabajo de revisión las investigaciones que analizan la IE, cualquiera que sea la perspectiva y/o el instrumento utilizado para su evaluación (para una revisión sobre los instrumentos de evaluación de la IE, ver O’Connor et al., 2019).

\section{Método}

\section{Protocolo y Registro}

La presente revisión sistemática de carácter teórico se ha llevado a cabo siguiendo los estándares habituales de PRISMA (versión PRISMA-P, Moher et al., 2015) que consiste en una lista de verificación compuesta por 17 ítems. La pregunta guía para la revisión sistemática ha sido: ‘Existe una relación empírica entre el vínculo de apego y la inteligencia emocional en población adulta?'

\section{Criterios de Elegibilidad}

Con relación a los criterios de inclusión de los registros, se incorporaron registros académicos que describiesen: a) investigaciones que examinaran la relación existente entre el apego y la inteligencia emocional, b) estudios que se realizaran con personas adultas (p.ej., mayores de 18 años), y c) trabajos publicados en castellano o en inglés.

Respecto a los criterios de exclusión, se descartaron: a) todos aquellos trabajos teóricos o conceptuales, tales como revisiones o editoriales o comentarios, b) los documentos que no fuesen artículos revisados por pares o tesis doctorales, c) redactados en idiomas diferentes a los dos designados para la selección, d) que no proporcionasen un instrumento de apego o de IE, o que a pesar de utilizarse ambos, no se presentara un estadístico relacionándolos, y e) cuyas muestras estuviesen compuestas por personas menores de edad. Finalmente, se descartaron aquellos artículos para los cuales no se tuviese disponibilidad de texto completo, ni desde las bases de datos correspondientes, ni a través de redes profesionales de personal académico (p.ej.,'ResearchGate'). 


\section{Fuentes de Información y Búsqueda}

La búsqueda sistemática de la literatura científica se llevó a cabo en las siguientes bases de datos electrónicas: Web of Science, PsycArticles, PsycInfo y Psicodoc. En la estrategia de búsqueda se utilizaron las palabras clave: 'emotional intelligence' y 'attachment' (así como sus equivalentes en castellano 'inteligencia emocional' y 'apego' para la base de datos Psicodoc). Ambos términos se buscaron de forma combinada mediante el conector booleano 'AND' y sin especificar ninguna categoría de campo (es decir, incluyendo todo el texto y/o descriptores de indexación) ni limitación temporal de publicación. La búsqueda tuvo lugar en octubre de 2020 por dos revisoras de manera independiente. Se añadieron manualmente registros identificados al que tuvieron acceso las personas autoras del artículo por medio de contactos personales.
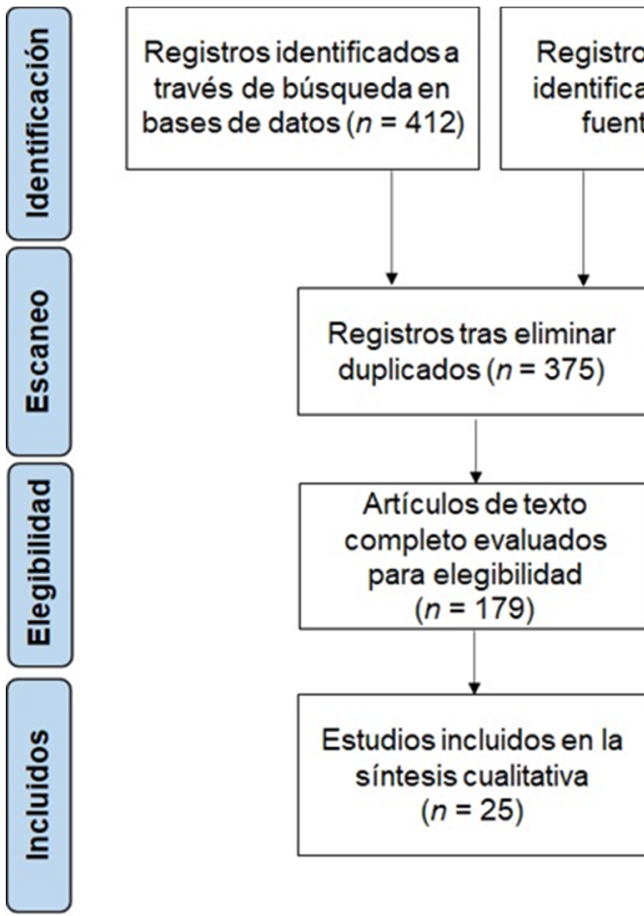

Figura 1.

Flujograma PRISMA de la Revisión Sistemática

\section{Codificación de los Estudios}

Una vez que se constató que los artículos mostraban una relación empírica entre el apego seguro y la inteligencia emocional, se analizó el contenido completo de los mismos con el fin de extraer de cada uno de ellos información relativa a determinados criterios para evaluar la calidad, criterios previamente consensuados entre las revisoras. Dicha información fue codificada en dos hojas de Excel para su posterior análisis y discusión. Los criterios elegidos fueron los siguientes: a) autoría, b) año de publicación, c) título, d)

\section{Selección de Estudios}

Se seleccionaron los artículos utilizados en la revisión del Diagrama de búsqueda PRISMA (Moher et al., 2015). La Figura 1 recoge el proceso de selección de los estudios. En la búsqueda inicial, tras aplicar la estrategia de búsqueda, se encontraron 420 potenciales estudios. A continuación, se eliminaron 45 artículos duplicados, y de los 375 restantes se seleccionaron 179 artículos en función del resumen para una valoración de su elegibilidad buscando la información en el texto completo. Se descartaron los estudios que no cumplían con los criterios de inclusión $(n=154)$, dando como resultado un total de 25 estudios que cubrían los requisitos para ser integrados en la revisión sistemática. 
Tabla 1.

Estudios y Características de los Registros incluidos en la Revisión Sistemática

\begin{tabular}{lcccl}
\hline Autores y año & $\begin{array}{c}\text { n, tipo de } \\
\text { muestra y \% } \\
\text { mujeres }\end{array}$ & $\begin{array}{c}\text { Tipo e instru- } \\
\text { mento de IE } \\
\text { usado }\end{array}$ & $\begin{array}{c}\text { Tipo e ins- } \\
\text { trumento de } \\
\text { apego usado }\end{array}$ & Resultados principales \\
\hline $\begin{array}{l}\text { Aguilar-Luzón } \\
\text { et al. (2012) }\end{array}$ & $\begin{array}{c}\text { (44) est. univ. } \\
(69,44 \%)\end{array}$ & $\begin{array}{c}\text { IEP: TMMS- } \\
\text { Dimensiones: }\end{array}$ & $\begin{array}{c}\text { La seguridad del apego se relaciona positivamente con } \\
\text { claridad. La evitación se relaciona negativamente con } \\
\text { claridad. La ansiedad se relaciona negativamente con } \\
\text { atención emocional. Los niveles más altos de regulación } \\
\text { emocional se dan en seguros (baja evitación y ansiedad). }\end{array}$
\end{tabular}

Anticevic et al. 77: 30 pacien(2019)

tes TLP y 47

control

$(100 \%)$

Cherry et al.

(2013)

200 est. univ. $(56 \%)$

IEC: MSCEIT 45 FAQ

Las dos dimensiones de apego inseguro se asocian $(-0,30$ $<r<-0,49)$ con las dimensiones de competencia emocional. La evitación muestra una asociación mayor con la expresión de emociones que la ansiedad.

Dimensiones: La evitación (apego) correlacionó con la IE $(-0,28)$, pero ECR-SF la ansiedad (apego) solo con IE experiencial-Percibir emociones y Facilitar pensamientos- $(0,19)$. Solamente la evitación predijo de manera significativa la IE, explicando un $13 \%$ de la varianza.

Cherry et al. 296 est. univ. IEC: MSCEIT Dimensiones: (2014) (55\%) ECR-SF

Cleveland y

Yu (2019)

284 est. univ. $(100 \%)$
IEP: TEIQ- ASF

Dimensiones:
ECR-RSQ

El apego evitativo correlaciona negativamente con la IE, pero el apego ansioso no. El modelo de ecuaciones estructurales mostró que el apego evitativo predijo de manera significativa y negativa la IE.

La IEP se relaciona negativamente con la inseguridad en el apego, siendo mayor el efecto para apego a pares ($0,32)$ que para apego a padres $(-0,19)$.

Dimitrijevic et al. (2018)

566: 288 población gene$\operatorname{ral}(44,4 \%)$; 278 est. univ. $(72,3 \%)$

Dimitrijevic et al. (2020)

251 empleados/as (53,78\%)

Mixta: TEIQue

Mixta:

Dimensiones: ECR-R IEC: MSCEIT v. 2.0

QAA-R

IEC: MSCEIT v. 2.0

Dimensiones: ECR-R

Dimensiones: QAA-R

Doba y Nan61 pacientes drino (2019) AN (100\%)

IEP: PEC

Dimensiones: ECR

Hamarta et al. (2009)

463 est. univ. $(58,7 \%)$

Mixta: EQ-I

Estilos: RSQ

Ambas dimensiones de inseguridad de apego muestran correlaciones negativas con IEC e IEM, siendo mayor en el caso de ansiedad; IEM (ansiedad: $-0,48$ y $-0,62$, evitación: $-0,40$ y -0,48), IEC (ansiedad: $-0,32$ y $-0,47$, evitación: $-0,07 \mathrm{y}-0,27)$.

La seguridad del apego se relaciona positivamente con IE (tanto IEC como IEP). El apego seguro (estilos) se asocia más fuertemente a IE (IEC e IEP) que cualquier apego inseguro. La relación con el apego es mayor con IEP que con IEC. Los mayores correlatos del apego fueron la capacidad para manejar emociones (IEC) y la Emocionalidad (IEP).

La inseguridad en el apego predice negativamente de manera indirecta la competencia emocional intrapersonal, pero solamente la evitación predice negativamente la competencia emocional interpersonal.

Hay asociaciones de 0,20 a 0,33 entre el estilo de apego seguro y todas las subescalas de IEC y del apego temeroso $\mathrm{y}$ todas las subescalas de IEC (entre $-0,10$ y $-0,21)$. El estilo evitativo $(-0,13)$ y el preocupado $(0,10)$ solo se asocian con la competencia interpersonal.

Iliceto y Fino 476 población (2017)

general

$(38,7 \%)$
IEP: WLEIS

Dimensiones: 9AP
La baja ansiedad y evitación se asocian a alta IE; sin embargo, el uso de la emoción solamente se asocia a la ansiedad. 
Tabla 1.

Estudios y Características de los Registros incluidos en la Revisión Sistemática (continuación)

\begin{tabular}{lccc}
\hline Autores y año & $\begin{array}{c}\boldsymbol{n} \text {, tipo de } \\
\text { muestra y \% } \\
\text { mujeres }\end{array}$ & $\begin{array}{c}\text { Tipo e instru- } \\
\text { mento de IE } \\
\text { usado }\end{array}$ & $\begin{array}{c}\text { Tipo e ins- } \\
\text { trumento de } \\
\text { apego usado }\end{array}$ \\
\hline Kafetsios & $\begin{array}{c}239 \text { población } \\
\text { general }\end{array}$ & IEC: MSCEIT & Estilos: RQ \\
$(2004)$ & $\begin{array}{c}\text { v. } 2.0 \\
\end{array}$ & &
\end{tabular}

Resultados principales

$\operatorname{Kim}(2005) \quad 115$ est. univ $(57,39 \%)$

Laible (2007)

117 est. univ. (56\%)

Lanciano et al. (2012)

\section{Li y Zheng}

(2014)

Marks et al. (2016)

Nandrino et al. (2020)

Neustadt et al. (2011)

Obeid et al. (2019)

585 est. univ. (46,15\%)

343: est. univ. y población general (78,13\%)

126: 63 pacientes con AN restrictiva y 63 controles (100\%)

124 trabajadores hoteles (\% 48)

789 población general $(45,25 \%)$

Páez et al. (2006)

357 est. univ. (? \%)

Ramos Mejia (2016)
157 población general (71,3\%)
IEP: TMMS

Dimensiones: AAQ

IEP: SEQ IEP: TAS-20

Dimensiones: IPPA

IEP: SREIT

IEP: SREIT

IEP: PEC

Dimensiones: ECR

Mixta: TEIQue

IEP: QEISA IEP: TAS-20

Dimensiones: AAW

Estilos: RQ

IEM se asocia a la seguridad en el apego $(0,55)$ e inseguridad $(-0,51)$. Hay una única dimensión de inseguridad. nor competencia intrapersonal, y solo la primera se asocia a menor competencia interpersonal. Ambas dimensiones del apego correlacionan negativamente con IEC: entre -0,29 y -0,35 para evitación; entre 0,18 y $-0,27$ para ansiedad. la emoción. El apego evitativo se asocia positivamente con la habilidad para comprender las emociones.

Existe asociación entre apego seguro e IE percibida (beta $=0,51)$. No hay asociación entre apego ansiosoambivalente e IE percibida.

La seguridad en el apego se asocia positivamente con expresividad emocional positiva $(0,31,0,31)$, conciencia emocional $(0,42,0,36)$, y negativamente con la expresividad emocional negativa $(-0,38,-0,24)$.

ECR

Las dos dimensiones de apego correlacionan negativamente con IE (ansiedad: -0,14; evitación: -0,28).

Las dos dimensiones de apego correlacionan negativamente con IE (ansiedad: -0,31; evitación: -0,40).

\section{ECR}

El estilo seguro correlaciona positivamente con todas las dimensiones de IEP (entre 0,19 y 0,27 ) y negativamente con alexitimia $(-0,11)$. Los estilos de apego inseguro correlacionan negativamente con IEP (entre - 0,08 y -0,13), y positivamente con alexitimia para el apego preocupado $(0,25)$.

IEP: TMMS24

IEP: TAS-20

IEC: MSCEIT v. 2.0

IEP: TMMS48
Estilos: Tipos de apego romántico

El apego seguro se asocia negativamente a IEP deficitario $(-0,19)$, formado por alexitimia y bajas claridad y regulación. El apego temeroso se asocia negativamente a IEC ($0,29)$ y positivamente a IEP deficitario $(0,38)$.

Estilos: Tipos de Apego Romántico
El apego ansioso (estilo) se asocia negativamente con la claridad de IEP (se reportan dos correlaciones distintas: $0,26$ y $-0,28)$. 
Tabla 1.

Estudios y Características de los Registros incluidos en la Revisión Sistemática (continuación)

\begin{tabular}{|c|c|c|c|c|}
\hline Autores y año & $\begin{array}{c}n, \text { tipo de } \\
\text { muestra y \% } \\
\text { mujeres }\end{array}$ & $\begin{array}{l}\text { Tipo e instru- } \\
\text { mento de IE } \\
\text { usado }\end{array}$ & $\begin{array}{c}\text { Tipo e ins- } \\
\text { trumento de } \\
\text { apego usado }\end{array}$ & Resultados principales \\
\hline $\begin{array}{l}\text { Rossen y } \\
\text { Kranzler } \\
(2009)\end{array}$ & $\begin{array}{l}150 \text { est. univ. } \\
(73,33 \%)\end{array}$ & $\begin{array}{l}\text { IEC: MSCEIT } \\
\text { v. } 2.0\end{array}$ & $\begin{array}{l}\text { Dimensio- } \\
\text { nes: IPPA }\end{array}$ & No se observa correlación entre apego hacia pares e IE. \\
\hline $\begin{array}{l}\text { Shorey y } \\
\text { Chaffin (2018) }\end{array}$ & $\begin{array}{c}95 \text { ejecutivos } \\
\text { de negocios } \\
(24,21 \%)\end{array}$ & Mixta: EQ-I & $\begin{array}{c}\text { Dimensio- } \\
\text { nes: ECR } \\
\text { Dimen- } \\
\text { siones: LERS }\end{array}$ & $\begin{array}{l}\text { El apego a los líderes se asocia negativamente a la IE, más } \\
\text { a la evitación }(-0,38) \text { que a la ansiedad }(-0,26) \text {. }\end{array}$ \\
\hline $\begin{array}{l}\text { Stevens } \\
(2017)\end{array}$ & $\begin{array}{l}116 \text { est. univ. } \\
(83,62 \%)\end{array}$ & $\begin{array}{l}\text { IEP: SREIT } \\
\text { IEP: TAS-20 }\end{array}$ & $\begin{array}{l}\text { Dimensio- } \\
\text { nes: ECR }\end{array}$ & $\begin{array}{l}\text { La evitación en el apego se asocia con alexitimia }(0,42) \text { y } \\
\text { negativamente con IE total y dimensiones (correlaciones } \\
\text { entre }-0,20 \text { y }-0,28) \text {. La ansiedad en el apego no se asocia a } \\
\text { la alexitimia ni a la IEP total, aunque correlaciona }(-0,19) \\
\text { con manejo de las emociones propias y con uso de la emo- } \\
\text { ción }(0,19) \text {. }\end{array}$ \\
\hline $\begin{array}{l}\text { Tyszkiewicz- } \\
\text { Bandur et al. } \\
(2017)\end{array}$ & $\begin{array}{l}328 \text { est. univ. } \\
\quad(76 \%)\end{array}$ & IEP: SREIT & Estilos: RQ & $\begin{array}{l}\text { Personas de apego seguro informan de más IEP que las de } \\
\text { apego inseguro. }\end{array}$ \\
\hline \multicolumn{5}{|c|}{$\begin{array}{l}\text { Nota. IE = inteligencia emocional, IEP = inteligencia emocional percibida, IEC = inteligencia emocional capacidades, AN = } \\
\text { Anorexia nerviosa, TLP = Trastorno Límite de Personalidad, TMMS = Trait Meta-Mood Scale, TEIQue = Trait Emotional } \\
\text { Intelligence Questionnaire, TEIQ-ASF = Trait Emotional Intelligence Questionnaire-Adolescent Short Form, MSCEIT = May- } \\
\text { er-Salovey-Caruso Emotional Intelligence Test, EQ-I = Bar-On Emotional Quotient Inventory, PEC = Profile of Emotional } \\
\text { Competence, SREIT = Self-Report Emotional Intelligence Test, QEISA = Quick Emotional Intelligence Self-Assessment, } \\
\text { WLEIS = Wong and Law Emotional Intelligence Scale, UEK = Emotional Competence Questionnaire, SEQ = Self- } \\
\text { Expresiveness Questionnaire, TAS-20 = Toronto Alexithymia Scale-20, ECR = Experiences in Close Relationships, ECR-R = } \\
\text { Experiences in Close Relationships-Revised, ECR-SF = Versión abreviada del Experiences in Close Relationships, ECR-RSQ } \\
=\text { Experiences in Close Relationships-Relationship Structures Questionnaire, RSQ = Relationships Scales Questionnaire, RQ } \\
\text { Relationship Questionnaire, QAA-R = Questionnaire for Attachment Assessment-Revised, AAQ = Adult Attachment Ques- } \\
\text { tionnaire, LERS = Leaders' Experiences in Relationships, 9AP =9 Attachment Profile, IPPA = Inventory of Parent and Peer } \\
\text { Attachment, AAW = Adult Attachment in the Workplace, FAQ = Friendship Attachment Questionnaire. }\end{array}$} \\
\hline
\end{tabular}

\section{Resultados}

Atendiendo a la pregunta general de este estudio, y tal y como se esperaba, se constata que existe una relación empírica entre el apego adulto y la IE. De los 25 artículos examinados, en 24 de ellos aparece una asociación en forma de estadístico que así lo atestigua, es decir, solamente en un único estudio no se observa tal relación. Con respecto a la metodología y la estrategia de análisis de datos, todos los estudios analizados utilizaron una metodología correlacional de encuesta para valorar la relación entre apego adulto e IE. Los estadísticos utilizados incluyen correlaciones en la mayoría de los casos (20 estudios), la prueba t para la comparación de medias en uno de ellos, proporcionándose en 15 estudios coeficientes de regresión para predecir la IE a partir del apego, a pesar de que no se utilizan diseños experimentales ni longitudinales.

Por otra parte, la relación existente entre apego e IE se ha confirmado con diferentes tipos de población. En concreto, en la presente revisión se aportan evidencias en 15 muestras de estudiantes universitarios, seis muestras de población general, tres muestras clínicas (dos de anorexia nerviosa y una de trastorno límite de personalidad) y tres muestras de trabajadores o trabajadoras.

Otro elemento del análisis lo constituye la existencia de países diferentes donde se ha observado la relación entre las variables a estudio de la presente revisión. A este respecto, existe una variedad considerable. En concreto, seis estudios se han llevado a cabo en EEUU (en el séptimo realizado en dicho país es el cual no se observó la relación), cuatro en el Reino Unido, dos en Francia, dos en Serbia, dos en Italia, uno en Turquía, uno en Líbano, uno en Croacia, uno en China, uno en Argentina, uno en Polonia, uno en España, y finalmente uno llevado a cabo con una muestra recogida y analizada de manera conjunta en España, Chile y México. 
Profundizando un poco más en la relación entre las variables, la misma resulta patente en estudios que evalúan la IE tanto desde la perspectiva de las capacidades como desde la autopercepción del propio individuo. En los dos estudios llevados a cabo en Serbia, se evaluó de manera conjunta las capacidades IE con el MSCEIT v.2.0 y la IE mixta autoinformada con el cuestionario TEIQue en dos muestras de empleados y en una de estudiantes universitarios; en ambos se observó que las asociaciones con el apego eran menores cuando se evaluaban las capacidades.

Con respecto a los estudios que evalúan las capacidades de la inteligencia emocional (IEC), en tres de ellos se examina su relación con un índice de apego. Si bien en dos de ellos se corrobora una asociación positiva con el apego seguro, en el tercero no se observa tal relación. Este último estudio, que evalúa el apego con un instrumento de evaluación de la seguridad en adolescentes (p.ej., IPPA), no proporciona correlaciones; la relación entre apego e IE se examina a través de un coeficiente de regresión en el que además se introducen más predictores. En lo que respecta a las dimensiones de inseguridad, en seis estudios se examina tal relación. La asociación negativa de la IEC con respecto a la dimensión de ansiedad resulta clara: en los seis estudios se informa de dicho resultado, e incluso en dos de ellos posteriormente se concluye que la ansiedad en el apego es un predictor que explica el constructo IE capacidades, incluso más allá de la personalidad y de la inteligencia. Sin embargo, la dimensión de evitación solamente muestra una relación negativa con las capacidades de IE en tres de esas seis investigaciones, evidenciando por tanto una relación menos estable o interdependiente de otras posibles variables asociadas.

Analizando el resto de los instrumentos de evaluación y desde una perspectiva de autopercepción, se constata la relación entre apego seguro y IEP. Teniendo en cuenta que las dimensiones que lo componen son diferentes, se examinan de manera independiente los estudios atendiendo al instrumento de evaluación. Así, de los cuatro estudios que utilizan el TMMS en alguna de sus versiones, dos de ellos proporcionan una puntuación compuesta o general de IEP, mostrando en ambos una asociación positiva con respecto a la seguridad en el apego. De estos cuatro estudios, no obstante, solamente tres de ellos proporcionan estadísticos para las dimensiones de Claridad Emocional (CE), Atención Emocional (AE) y Regulación Emocional (RE); en dos de ellos se informa de una asociación positiva entre seguridad en el apego y tanto CE como RE, así como de la ausencia de relación con $\mathrm{AE}$. Con respecto a las dos orientaciones de inseguridad en el apego, conviene señalar que dos de los tres estudios han utilizado únicamente una medida continua de los tres estilos de apego, por lo que el valor de apego ansioso se refiere a un perfil de alta ansiedad y baja evitación, y el evitativo no distingue entre las dos estrategias de evitación subyacentes. Así, considerando este estilo ansioso/ambivalente, en esos dos estudios se constata una relación negativa con respecto a CE. El tercer estudio, que considera la ansiedad desde una perspectiva bidimensional y distingue también los cuatro patrones, no revela relación alguna con la CE, si bien desde la primera perspectiva la tendencia es en ese sentido ( $r=-0,15$, n.s.). En este último estudio, desde la perspectiva bidimensional ortogonal ansiedad y evitación-, se observa una relación negativa de la CE con la evitación en el apego, así como una asociación positiva de la AE con la ansiedad en el apego.

Por otra parte, cuatro de los estudios revisados incluyen también un instrumento que evalúa la dificultad para identificar y describir sentimientos o alexitimia (TAS-20, Bagby et al., 1994). Los resultados proceden de investigaciones que utilizan instrumentos de apego que parten de la concepción bidimensional, evaluación dimensional de tres estilos o incluso unidimensional (continuum seguridad vs. inseguridad en el apego). En tres de ellos se evidencia la asociación negativa entre tal dificultad emocional y el apego seguro. En el cuarto estudio —el único con medida bidimensional ortogonal del apego donde se distinguen claramente las dos orientaciones de inseguridad en el apegose observa una relación positiva únicamente con la dimensión de evitación, lo cual es compatible con los presupuestos teóricos.

Hay dos estudios revisados que emplean el instrumento PEC (Brasseur et al., 2013), observándose en ambos los mismos resultados que se describen a continuación: por un lado, la competencia intrapersonal muestra una relación negativa con las dos dimensiones de inseguridad en el apego - ansiedad y evitación-; por otro lado, la competencia interpersonal, tal y como sería esperable, solamente se asocia de manera negativa a la dimensión de evitación.

Cuatro investigaciones han examinado la cuestión utilizando el instrumento SREIT (Schutte et al., 1998). En todas ellas se proporciona información de la relación entre apego e IE total: en un estudio, tras dicotomizar la muestra entre personas con apego seguro e inseguro, se informa de mayores puntuaciones de IE para la submuestra de seguros; en otros dos estudios se constata que las dos dimensiones de inseguridad en el apego correlacionan negativamente con IE, siendo mayor en el caso de la evitación $(r=-0,28 \mathrm{y}-$ $0,40)$ en comparación con la ansiedad $(r=-0,14$ y $-0,31)$; en el cuarto estudio, con una muestra menor de estudiantes universitarios, se confirma solamente la asociación negativa entre IE y evitación ( $r=-0,28)$, no existiendo para la dimensión de ansiedad, donde no alcanza significación estadística ( $r=0,04$, n.s.). Solamente este cuarto estudio analiza también la relación entre apego e IE al nivel de factores; la evitación se relaciona negativamente con los cuatro factores del SREIT, si bien en Uso de emociones no 
alcanza significación estadística (la muestra tiene una $n$ de 116); con respecto a la dimensión de ansiedad, únicamente los factores Uso de emociones $(r=0,19)$ y Manejo de las emociones propias $(r=-0,19)$ se asocian a la misma.

Finalmente, otros tres estudios han proporcionado evidencia de la relación positiva entre seguridad del apego e IE global evaluada con el instrumento TEIQue (Petrides, 2009); una cuarta investigación, además, distingue entre las dos dimensiones de inseguridad del apego, mostrando asociaciones negativas de magnitud medio-alta, tanto con ansiedad como con evitación, para las cuatro dimensiones de IE - bienestar, autocontrol, emocionalidad y sociabilidad - ( $r$ s entre $-0,34$ y $-0,62)$.

\section{Discusión}

El objetivo de este trabajo era realizar una revisión sistemática teórica que describiese de manera resumida los hallazgos que relacionaban de manera empírica apego e IE. Un considerable número de artículos revisados por pares constata la existencia de una relación entre la seguridad en el apego con la IE y varias de sus dimensiones.

Un aspecto destacable es la constatación de dicha relación en un número considerable de culturas. Ello es importante porque aporta evidencias de la universalidad de dicha relación, al tiempo que se huye del posible anglocentrismo en la publicación en ciencias sociales al que se aludía y que estaba en el centro del debate a finales del siglo XX (p.ej., MacCoun, 1998; Wierzbicka, 1993). Ciertamente la adaptación rigurosa de los instrumentos de evaluación de IE a diferentes culturas ha posibilitado el desarrollo científico en el área emocional -MSCEIT en versión serbia (Altaras -Dimitrijević y Jolić-Marjanović, 2010) o italiana (D’Amico y Curci, 2011); TMMS-24 en versión española (Fernández-Berrocal et al., 2004) o SREIT en versión china (Zheng et al., 2009) -

Las investigaciones examinadas en esta revisión se enmarcan en diferentes áreas y emplean muestras de procedencia diversa (población general, clínica y organizacional). Así, a pesar de que las dimensiones originales del apego adulto se refieren al contexto de las relaciones de pareja (Brennan et al., 1998), las diferencias individuales en dichas dimensiones pudieran ser aplicables a diferentes ámbitos, aunque con otros matices, en los que una figura pueda proporcionar seguridad interpersonal. Este es el caso del ámbito organizacional, donde se han producido reformulaciones de los instrumentos para adaptarlos al contexto (p.ej., Shorey y Chaffin, 2018).

El diseño y la metodología utilizados para comprobar las relaciones entre variables es en todos los casos transversal y los análisis empleados en gran medida combinan correlaciones con coeficientes de regresión. En varios casos se aplican modelos de ecuaciones estructurales que asumen una direccionalidad. Este posicionamiento común en los estudios revisados plantea el apego como antecedente de las capacidades emocionales. Sin embargo, la utilización de una estrategia de análisis que asume causalidad en estudios de corte transversal es una cuestión que siempre genera controversia en nuestro ámbito. La asunción teórica de que las dimensiones o estilos de apego preceden evolutivamente al surgimiento de capacidades de inteligencia emocional y, por tanto, pueden explicarlas (p.ej., Marks et al., 2016; Nandrino et al., 2020; Stevens, 2016) pudiera ser un argumento válido para proponer y testar modelos de este tipo. El origen de la vinculación afectiva en la infancia y su continuidad o estabilidad hacia la adultez estaría en la base del planteamiento, reforzado por el hecho de que la asociación entre apego inseguro vs. seguro en la infancia y su estabilidad en la adultez se demostró en el meta-análisis de Pinquart et al., (2013). No obstante, parece necesario que en este ámbito se puedan plantear estudios longitudinales que tracen el desarrollo preciso de las competencias de IE.

Queremos señalar, asimismo, algunas posibles limitaciones y/o posibles líneas de futuro del trabajo aquí descrito. Por un lado, la estrategia de búsqueda ha incluido únicamente dos términos; el uso generalizado de dichos términos en la indización en las bases de datos, así como su localización inespecífica (p.ej., en cualquier parte del documento), nos lleva a pensar que se ha podido asegurar una recuperación exitosa de los registros pertinentes. Por otro lado, si bien el análisis presentado en esta revisión sistemática proporciona un punto de partida para comprender qué datos disponemos con respecto a la relación entre el apego y la IE en la adultez, la variabilidad debida a factores asociados (es decir, posibles moderadores) no ha sido inspeccionada en el presente trabajo.

Este nivel de análisis más fino requiere de una aproximación cuantitativa o revisión meta-analítica, donde, además de cuantificar el tamaño del efecto de la relación entre las variables, se examinen también dichas variables moderadoras. En primer lugar, y con respecto al tipo de documento, pudiera darse un sesgo de publicación que convendría examinar. A este respecto, Aguilar-Luzón y colaboradoras (2012) señalaron en su revisión de la literatura que, mientras que en artículos revisados por pares se sugiere que el estilo de apego predecía las diferencias individuales en inteligencia emocional, en tres tesis doctorales analizadas por dichas autoras tal relación no se constataba. En segundo lugar, el género sería, sin duda, una de las variables que pudiera ejercer un efecto diferencial. Así, cabe señalar, por ejemplo, que en un meta-análisis de diferencias de género en IE (Joseph y Newman, 2010) se observó que, si bien en IE percibida las diferencias eran mínimas, con respecto a las capacidades, las mujeres percibían, comprendían, utilizaban y regulaban mejor las emociones. Otra posible variable mediadora a incluir sería el instrumento utilizado para 
medir IE, con la conceptualización que recoja, y sus posibles dimensiones, además de la propia distinción entre IE percibida o capacidades IE. En este sentido, en la presente revisión solamente en dos estudios (Dimitrijevic et al., 2018, 2020) se han utilizado simultáneamente los instrumentos TEIQue y MSCEIT, por lo que las diferentes magnitudes en la asociación con el apego requerirían de mayores evidencias.

En conclusión, a través de esta sistematización de las interrelaciones entre apego e IE se ha pretendido proporcionar un acercamiento inicial a la confluencia entre estos marcos teóricos. No solamente han generado a lo largo de estos 30 años un considerable avance de conocimiento teórico y aplicado de manera independiente, sino que las investigaciones que proporcionan más datos sobre cómo se relacionan apego e IE ayudan a completar el entramado explicativo de posibles variables contribuyentes al desarrollo de las capacidades de IE.

\section{Referencias}

Las referencias marcadas con asterisco corresponden a aquellas que contienen los estudios integrantes de la revisión.

*Aguilar Luzón, M., Calvo Salguero, A. y Monteoliva Sánchez, A. (2012). La inteligencia emocional percibida y su relación con el apego adulto. Psicología Conductual, 20(1), 119-135.

Ainsworth, M.D., Blehar, M., Waters, E. y Wall, S. (1978). Patterns of attachment: A psychological study of the Strange Situation. Erlbaum.

Alonso-Arbiol, I., Balluerka, N. y Shaver, P. R. (2007). A Spanish version of the Experiences in Close Relationships (ECR) adult attachment questionnaire. Personal Relationships, 14, 45-63. https:// doi.org/10.1111/j.1475-6811.2006.00141.x

Altaras-Dimitrijević, A. y Jolić-Marjanović, Z. (2010). The Mayer-Salovey-Caruso emotional intelligence test: Psychometric properties of the Serbian version. Psihologija, 43(4), 411-426. https://doi.org/10.2298/ PSI1004411A

*Antičević, V., Šošić, S. y Britvić, D. (2019). Emotional competence and coping mechanisms in Croatian women with Borderline personality disorder: The role of attachment. Psychiatria Danubina, 31(1), 8894. https://doi.org/10.24869/psyd.2019.88

Bagby, R.M., Parker, J.D. y Taylor, G.J. (1994). The twenty-item Toronto Alexithymia Scale-I. Item selection and cross-validation of the factor structure. Journal of Psychosomatic Research, 38(1), 23-32. https://doi.org/10.1016/0022-3999(94)90005-1
Bartholomew, K. (1990). Avoidance of intimacy: An attachment perspective. Journal of Social and Personal Relationships, 7, 147-178. https:// doi.org/10.1177/0265407590072001

Bartholomew, K. y Horowitz, L.M. (1991). Attachment styles among young adults: A test of a four-category model. Journal of Personality and Social Psychology, 61(2), 226-244. https://doi.org/10.1037/00223514.61.2.226

Bowlby, J. (1969/1982). Attachment and loss: Vol. 1. Attachment. Basic Books.

Brasseur, S., Grégoire, J., Bourdu, R. y Mikolajczak, M. (2013). The profile of emotional competence (PEC): Development and validation of a self-reported measure that fits dimensions of emotional competence theory. PloS one, 8(5), e62635. https:// doi.org/10.1371/journal.pone.0062635

Brennan, K.A., Clark, C.L. y Shaver, P.R. (1998). Selfreport measurement of adult attachment: An integrative overview. En J. A. Simpson y W. S. Rholes (Eds.), Attachment theory and close relationships (pp. 46-76). Guilford Press.

Cassidy, J. (2008). The nature of the child's ties. En J. Cassidy y P.R. Shaver (Eds.), Handbook of attachment. Theory, research, and clinical applications (2nd ed.), (pp. 3-22). The Guilford Press.

Cassidy, J. y Kobak, R.R. (1988). Avoidance and its relationship with other defensive processes. En J. Belsky y T. Nezworski (Eds.), Clinical implications of attachment (pp. 300-323). Erlbaum.

*Cherry, M.G., Fletcher, I. y O’Sullivan, H. (2013). Exploring the relationships among attachment, emotional intelligence and communication. Medical Education, 47(3), 317-325. https://doi.org/10.1111/ medu. 12115

*Cherry, M.G., Fletcher, I. y O'Sullivan, H. (2014). Validating relationships among attachment, emotional intelligence and clinical communication. Medical Education, 48(10), 988-997. https://doi.org/10.1111/ medu. 12526

*Cleveland, E.S. y Yu, M. (2019). Modeling female social aggression: predictors from multiple developmental contexts. The Journal of Genetic Psychology, 180(45), 185-204. https:// doi.org/10.1080/00221325.2019.1632254

Collins, N.L. y Read, S.J. (1990). Adult attachment, working models, and relationship quality in dating couples. Journal of Personality and Social Psychology, 58(4), 644-663. https://doi.org/10.1037/0022- 


\subsubsection{4}

D'Amico, A. y Curci, A. (2011). Traduzione ed adattamento italiano del Mayer-Salovey-Caruso Emotional Intelligence Test (MSCEIT) [Italian translation of the Mayer-Salovey-Caruso Emotional Intelligence Test (MSCEIT)]. OS.

*Dimitrijević, A., Hanak, N., Altaras Dimitrijević, A. y Jolić Marjanović, Z. (2018). The Mentalization Scale (MentS): A self-report measure for the assessment of mentalizing capacity. Journal of Personality Assessment, 100(3), 268-280. http:// dx.doi.org/10.1080/00223891.2017.1310730

*Dimitrijević, A.A., Marjanović, Z.J. y Dimitrijević, A. (2020). A further step towards unpacking the variance in trait and ability emotional intelligence: The specific contribution of attachment quality. Current Psychology, 39(4), 1340-1353. https:// doi.org/10.1007/s12144-018-9837-3

*Doba, K. y Nandrino, J.L. (2020). Cognitive and Emotional Empathy in Anorexia Nervosa: The Role of Attachment Insecurity, Intrapersonal, and Interpersonal Emotional Competences. The Journal of Nervous and Mental Disease, 208(4), 312-318. https:// doi.org/10.1097/NMD.0000000000001130

Extremera, N., Fernández-Berrocal, P. y Salovey, P. (2006). Spanish version of the Mayer-SaloveyCaruso Emotional Intelligence Test (MSCEIT). Version 2.0: Reliabilities, age and gender differences. Psicothema, 18, Suppl, 42-48.

Feeney, J. y Noller, P. (2001). Apego adulto. Desclée de Brouwer.

Fernández-Berrocal, P., Extremera, N. y Ramos, N. (2004). Validity and reliability of the Spanish modified version of the Trait Meta-Mood Scale. Psychological Reports, 94(3), 751-755. https://doi.org/10.2466/ pr0.94.3.751-755

Fraley, R.C. y Waller, N.G. (1998). Adult attachment patterns: A test of the typological model. En J. A. Simpson y W. S. Rholes (Eds.), Attachment theory and close relationships (pp. 77-114). Guilford Press.

Gillath, O., Karantzas, G.C. y Fraley, R.C. (2016). Adult attachment: A concise introduction to theory and research. Academic Press.

Gregory, M., Kannis-Daymand, L. y Sharman, R. (2019). A review of attachment-based parenting interventions: Recent advances and future considerations. Australian Journal of Psychology, 71, 1-14. https:// doi.org/10.1111/ajpy.12270.
*Hamarta, E., Deniz, M. y Saltali, N. (2009). Attachment styles as a predictor of emotional intelligence. Educational Sciences: Theory and Practice, 9(1), 213229.

Hazan, C. y Shaver, P. (1987). Romantic love conceptualized as an attachment process. Journal of Personality and Social psychology, 52(3), 511. https:// doi.org/10.1037/0022-3514.52.3.511

*Iliceto, P. y Fino, E. (2017). The Italian version of the Wong-Law Emotional Intelligence Scale (WLEISI): A second-order factor analysis. Personality and Individual Differences, 116, 274-280. http:// dx.doi.org/10.1016/j.paid.2017.05.006

Joseph, D.L. y Newman, D.A. (2010). Emotional intelligence: An integrative meta-analysis and cascading model. Journal of Applied Psychology, 95(1), 5478. https://doi.org/10.1037/a0017286

*Kafetsios, K. (2004). Attachment and emotional intelligence abilities across the life course. Personality and Individual Differences, 37, 129-145. https:// doi.org/10.1016/j.paid.2003.08.006

*Kim, Y. (2005). Emotional and cognitive consequences of adult attachment: The mediating effect of the self. Personality and Individual Differences, 39, 913923. https://doi.org/10.1016/j.paid.2005.03.010

*Laible, D. (2007). Attachment with parents and peers in late adolescence: Links with emotional competence and social behavior. Personality and Individual Differences, 43, 1185-1197. https://doi.org/10.1016/ j.paid.2007.03.010

*Lanciano, T., Curci, A., Kafetsios, K., Elia, L. y Zammuner, V.L. (2012). Attachment and dysfunctional rumination: The mediating role of emotional intelligence abilities. Personality and Individual Differences, 53, 753-758. http://dx.doi.org/10.1016/ j.paid.2012.05.027

*Li, X. y Zheng, X. (2014). Adult attachment orientations and subjective well-being: Emotional intelligence and self-esteem as moderators. Social Behavior and Personality: An International Journal, 42(8), 12571265. http://dx.doi.org/10.2224/sbp.2014.42.8.1257

MacCoun, R.J. (1998). Biases in the interpretation and use of research results. Annual Review of Psychology, 49(1), 259-287. https://doi.org/10.1146/ annurev.psych.49.1.259

*Marks, A.D., Horrocks, K.A. y Schutte, N.S. (2016). Emotional intelligence mediates the relationship between insecure attachment and subjective health outcomes. Personality and Individual Differences, 
98, 188-192. $\quad$ http://dx.doi.org/10.1016/ j.paid.2016.03.038

Mayer, J.D., Salovey, P. y Caruso, D.R. (2002). MayerSalovey-Caruso Emotional Intelligence Test: User's manual. MHS.

Mikulincer, M. y Shaver, P.R. (2007). Attachment in adulthood: Structure, dynamics, and change. Guilford Press.

Mikulincer, M. y Shaver, P.R. (2016). Adult attachment and emotion regulation. En J. Cassidy y P. R. Shaver (Eds.), Handbook of Attachment: Theory, research, and clinical applications (3r ed., pp. 507533). Guilford Press.

Mikulincer, M. y Shaver, P.R. (2019). Attachment orientations and emotion regulation. Current Opinion in Psychology, 25, 6-10.

Moher, D., Shamseer, L., Clarke, M., Ghersi, D., Liberati, A., Petticrew, M., ... PRISMA-P Group (2015). Preferred reporting items for systematic review and meta-analysis protocols (PRISMA-P) 2015 statement. Systematic Reviews, 4(1), 1. https:// doi.org/10.1186/2046-4053-4-1

*Nandrino, J.L., Dodin, V., Cottencin, O. y Doba, K. (2020). Effect of intrapersonal emotional competences on the relationship between attachment insecurity and severity of eating disorder symptoms in patients with restrictive anorexia. Journal of Clinical Psychology, 76(3), 476-492. https:// doi.org/10.1002/jclp. 22877

*Neustadt, E.A., Chamorro-Premuzic, T. y Furnham, A. (2011). Attachment at work and performance. Attachment \& Human Development, 13(5), 471-488. https://doi.org/10.1080/14616734.2011.602254

*Obeid, S., Haddad, C., Akel, M., Fares, K., Salameh, P. y Hallit, S. (2019). Factors associated with the adults' attachment styles in Lebanon: The role of alexithymia, depression, anxiety, stress, burnout, and emotional intelligence. Perspectives in Psychiatric Care, 55(4), 607-617. https://doi.org/10.1111/ ppc. 12379

O’Connor, P.J., Hill, A., Kaya, M. y Brett, M. (2019). The measurement of emotional intelligence: A critical review of the literature and recommendations for researchers and practitioners. Frontiers in Psychology, 10, $116 . \quad$ https://doi.org/10.3389/ fpsyg.2019.01116

*Páez, D., Fernández, I., Campos, M., Zubieta, E. y Casullo, M. (2006). Apego seguro, vínculos parentales, clima familiar e inteligencia emocional: socializa- ción, regulación y bienestar. Ansiedad y Estrés, $12(2$ $-3), 329-341$.

Petrides, K.V. (2009). Technical manual for the Trait Emotional Intelligence Questionnaires (TEIQue). London Psychometric Laboratory.

Pinquart, M., Feußner, C. y Ahnert, L. (2013). Metaanalytic evidence for stability in attachments from infancy to early adulthood. Attachment \& Human Development, 15(2), 189-218. http:// dx.doi.org/10.1080/14616734.2013.746257

*Ramos Mejía, G. (2016). Teorías implícitas sobre el amor y factores asociados. Un estudio con pacientes con dependencia afectiva patológica y población general residente en la Ciudad Autónoma de Buenos Aires. Psicodebate: Psicología, Cultura y Sociedad, 16(2), 9-34. http://dx.doi.org/10.18682/pd.v16i2.592

Ran, G. y Zhang, Q. (2018). The neural correlates of attachment style during emotional processing: An activation likelihood estimation metaanalysis. Attachment \& Human Development, 20(6), 626-633. https:// doi.org/10.1080/14616734.2018.1465105

*Rossen, E. y Kranzler, J.H. (2009). Incremental validity of the Mayer-Salovey-Caruso Emotional Intelligence Test Version 2.0 (MSCEIT) after controlling for personality and intelligence. Journal of Research in Personality, 43(1), 60-65. https://doi.org/10.1016/ j.jrp.2008.12.002

Salovey, P. y Mayer, J.D. (1990). Emotional intelligence. Imagination, Cognition, and Personality, 9, 185211. https://doi.org/10.2190/DUGG-P24E-52WK$6 \mathrm{CDG}$

Schutte, N.S., Malouff, J.M., Hall, L.E., Haggerty, D.J., Cooper, J.T., Golden, C.J. y Dornheim, L. (1998). Development and validation of a measure of emotional intelligence. Personality and Individual Differences, 25, 167-177. https://doi.org/10.1016/S01918869(98)00001-4

*Shorey, H.S. y Chaffin, J.S. (2018). Leader-Follower Attachment: Implications for Personality Assessment in Organizational Contexts. Journal of Personality Assessment, 100(5), 518-528. https:// doi.org/10.1080/00223891.2018.1472100

*Stevens, F.L. (2017). Authenticity: A mediator in the relationship between attachment style and affective functioning. Counselling Psychology Quarterly, 30(4), 392-414. http:// dx.doi.org/10.1080/09515070.2016.1176010

*Tyszkiewicz-Bandur, M., WalkieWicz, M., Tartas, M. y 
Bankiewicz-Nakielska, J. (2017). Emotional intelligence, attachment styles and medical education. Family Medicine \& Primary Care Review, 19(4), 404-407. https://doi.org/10.5114/fmpcr.2017.70127

West, M. y Sheldon-Keller, A.E. (1994). Patterns of relating. Guilford.

Wierzbicka, A. (1993). A conceptual basis for cultural psychology. Ethos, 21(2), 205-231. https:// doi.org/10.1525/eth.1993.21.2.02a00040

Woodhouse, S., Ayers, S. y Field, A.P. (2015). The relationship between adult attachment style and posttraumatic stress symptoms: A meta-analysis. Journal of Anxiety Disorders, 35, 103-117. https:// doi.org/10.1016/j.janxdis.2015.07.002

Zheng, X., Gu, H. y Zhu, P. (2009). A study of the Emotional Intelligence Scale (EIS) by the Multivariate Generalizability Theory [In Chinese]. Psychological Science, 32, 181-183.

Zheng, L., Luo, Y. y Chen, X. (2020). Different effects of attachment anxiety and attachment avoidance on depressive symptoms: A meta-analysis. Journal of Social and Personal Relationships, 37(12), 30283050. https://doi.org/10.1177/0265407520946482 